\title{
Non-spherical Gold Nanoparticles: Tumor Imaging and Therapy
}

Yunsheng Chen ${ }^{1,2}$, Daxiang Cui ${ }^{1,2^{*}}$

${ }^{1}$ School of biomedicine engineering, Shanghai JiaoTong University, Shanghai, 200240, China

${ }^{2}$ Department of Bio-Nano Science and Engineering, National Key Laboratory of Micro/Nano Fabrication Technology Institute of Micro \& Nano Science and Technology, Shanghai JiaoTong University, Shanghai, 200240, China

* Corresponding author: D.X. Cui (dxcui@sjtu.edu.cn)

\begin{abstract}
Non-spherical Gold Nanoparticles (AuNPs), with unique geometries properties, have become the new exciting focus of applications in tumor imaging and therapy recently. In this review, we summarized the properties and applications of non-spherical AuNPs for cancer imaging and therapy. We review four typical shapes of non-spherical AuNPs from their applications and advantages with the great optimism to the applications of non-spherical AuNPs in medicine.
\end{abstract}

Keywords: Non-spherical Gold Nanoparticles, Tumor Imaging, Therapy, Gold nanorods, Gold nanoprisms, Gold nanostars, Gold nanocages

Citation: Y.S. Chen and D.X. Cui, Non-spherical Gold Nanoparticles: Tumor Imaging and Therapy, Nano Biomed. Eng. $2013,5(4)$, 160-167. DOI: 10.5101/nbe.v5i4.p160-167.

\section{Introduction}

Cancer is one of the main causes of mortality all over the world, and the number of new cancer patients increases every year [1]. In past 30 years, the diagnosis and treatments have made rapid advances, but the overall suprvival rate from cancer has not improved substantially. Therefore, the accurate early-diagnosis and targeted therapies, two major challenges in the battle against cancer, are given much attention [2]. Recently, nanomaterials offer new opportunities for cancer diagnosis and treatment, because of their unique physicochemical properties, such as ultrasmall size, large surface area to mass ratio, and high reactivity. These properties can be used to overcome some of the limitations found in traditional therapeutic and diagnostic agents $[3,4]$. According to the National Cancer Institute (NCI), nanomaterials have tremendous potential to make an important contribution in cancer prevention, diagnosis, imaging, and treatment $[5,6]$. Several materials are most used for cancer nanotechnology include polymers, dendrimers, liposomes, perfluorocarbons, quantum dots, iron oxides, nanotubes, nanowires and gold nanoparticles (AuNPs) [7-9]. In particular, AuNPs has been considered as the ideal nano-objects for cancer imaging and therapy, and been the subject of considerable recent studies.

In general, AuNPs can be classified as spherical and non-spherical particles [10]. Non-spherical AuNPs, such as rods, cages, prisms, stars or other structures, have received significant attention from researchers because of their structures. Our group dedicates to applications of various non-spherical AuNPs in cancer imaging and therapy. In this short review, we will summarize the properties and applications of non-spherical AuNPs for cancer imaging and therapy.

\section{The properties of non-spherical AuNPs}

\subsection{Optical properties}

The most interesting optical property of non-spherical AuNPs is the presence of multiple absorption bands correlated with their multiple axes [9,11-14]. The ease of tuning their optical properties gradually with particle size and shape makes them very interesting. Typically, anisotropic gold nanomaterials, such as rod-, prism-, star- and cage-like AuNPs of an appropriate size, among others, display a surface plasmon resonance (SPR) band in the near-infrared (NIR) region of the electromagnetic spectrum $[15,16]$. Importantly, tissue penetration of radiation can be improved (up to a few centimeters) by minimizing photon absorption by tissue components, which is typically achieved by tuning the SPR band from the visible to the NIR range [17]. Combining with biocompatibility of gold, non-spherical AuNPs show the great potential in cancer imaging. 


\subsection{Optothermal properties}

In addition to the enhanced and tunable optical properties for optical imaging, the strong optical absorption behavior of AuNPs is capable of creating a photothermal effect [18-20]. AuNPs are very photostable and biocompatible, which make them a new generation photothermal contrast agents for photothermal therapy (PTT) in which photon energy is converted to heat sufficient to induce cellular damage via thermal effects such as hyperthermia, coagulation and evaporation $[21,22]$. Generally, the optothermal conversion efficiency can be turned on selectively by tuning the wavelength to match that of the SPR of AuNPs. Non-spherical AuNPs possess several interesting light-absorbing properties, such as their extremely large photo-absorption cross sections and remarkably sensitive spectral shifts of their SPR so they have the excellent optothermal property $[21,23,24]$.

\subsection{Structural properties}

Compared to spherical AuNPs, non-spherical AuNPs show some structural properties for their specific geometry. They own more active surface sites to conjugate more targeting molecules, which provides the excellent selectivity of recognizing tumor cell $[25,26]$. They also possess superior long blood circulation time due to the anisotropic geometries, which can improve their targeting effect [27]. Furthermore, the stars- and cages-like AuNPs provide the enough space to load drug and be applied to targeting drug-delivery system, which has been considered as the assist of photothermal therapy (PTT) [28-30].

\section{Application of non-spherical AuNPs}

It is well known that the shape of AuNPs strongly influences their applicability to certain domains. Several synthetic protocols have been developed to fabricate different geometry AuNPs, such as rod-, star-, prisms- and cage-like particle. Meanwhile, the non-spherical AuNPs have attracted much attention for biomedical applications, including imaging techniques and PTT. Different shapes have different advantages and disadvantages, so we review four typical shapes non-spherical AuNPs in follows.

\subsection{Gold nanorods (GNRs)}

NGRs are considered as the simplest shape among in various non-spherical AuNPs. They have relatively facile synthetic procedure making them more feasible for future clinic settings [31]. GNRs have the strong longitudinal SPR band in NIR region with the different aspect ratio, which can improve tumor imaging and PPT [32,33].

GNRs are usually produced by surfactant cetyltrimethyl ammonium bromide (CTAB) directed synthesis, which produces a high yield of homogeneous GNRs with SPR resonances in the visible and NIR region [34]. In this approach, CTAB is not only the structure-directing agent of anisotropic growth, but also the stabilizer that forms bilayers on the GNRs. The CTAB-caped GNRs exhibit the significant cytotoxicity, but removing CTAB from the surface may cause the uncontrollable aggregation of nanoparticle [35-38]. Our group uses polyamidoamine dendrimer to replace CTAB molecules on the surface of gold nanorods by round-trip phase transfer ligand exchange [39]. When the resultant dendrimer-modified gold nanorods conjugated with arginine-glycine-aspartic acid (RGD) peptides, they exhibit very low cytotoxicity and highly selective targeting and destructive effects on the cancer cells under NIR laser irradiation. As shown in Fig. 1, Melanoma A375 cells incubated with RGDdGNR exhibit a strong golden color, while the melanoma A375 cells incubated with free RGD peptides and preincubated with free RGD peptides do not exhibit any golden color. This finding suggests that RGD-dGNR can specifically target to the surface and cytoplasm of tumor cell. Furthermore, the potential application of RGDdGNR in PTT is also investigate by using Mouse models loaded with melanoma A375 cells. The results incadute that RGD-dGNRs had the selective PTT to tumor cells on the tumor locations under the the NIR laser irradiation.

As the same time, GNRs also can be applied to enhanced radiation therapy effects. Our group uses silica to replace CTAB molecules on the surface of GNRs and anchor folic acid (FA) molecule [40]. FA-Si-GNRs exhibit highly selective targeting, enhanced radiation therapy (RT) and PTT effects on cancer cells using MGC803 cells in Fig. 2. As shown in Fig. 3, the corresponding injection site of mice displays a clearly distinguished CT signal and $\mathrm{CT}$ imaging in vivo. Based on our results, the dual-mode RT and PTT monitored by CT imaging could be achieved and bring novel opportunities in theranostics.
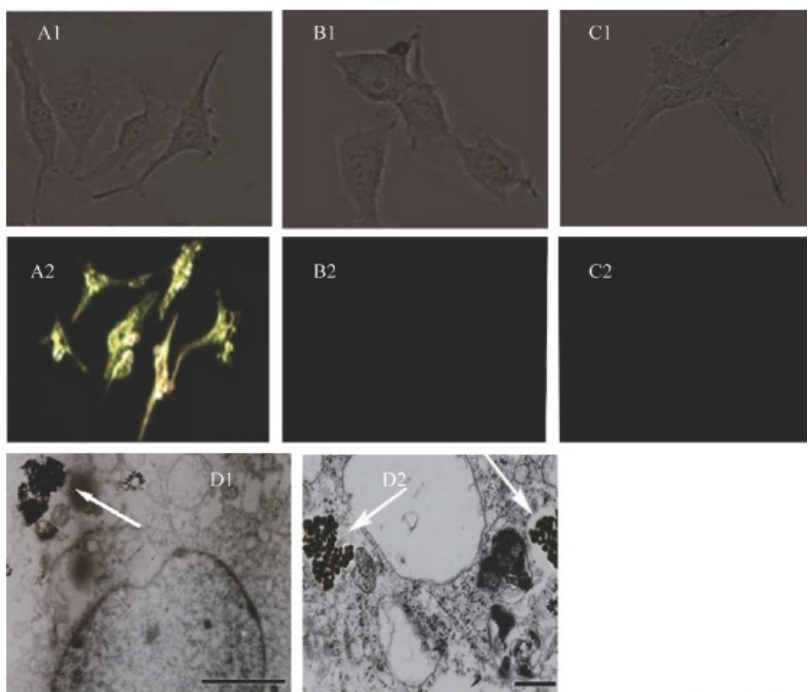

Fig. 1 Light scattering images and intracellular location of RGDdGNRs. Reflective mode dark-field images and bright field images of RGD-dGNRs (A), dGNRs (B), and free peptide and RGD-dGNRs (C) after incubation with A375 cells for $30 \mathrm{~min}$ at room temperature. A1, B1 and $\mathrm{C} 2$ are the bright field images of corresponding A375 cells. D1 and D2 are TEM images of RGD-dGNRs (arrow) in cytoplasm of melanoma A 375 cells, left scale bar, $500 \mathrm{~nm}$; right scale bar, $200 \mathrm{~nm}$. Reprinted with permission from Ref. 39 . 

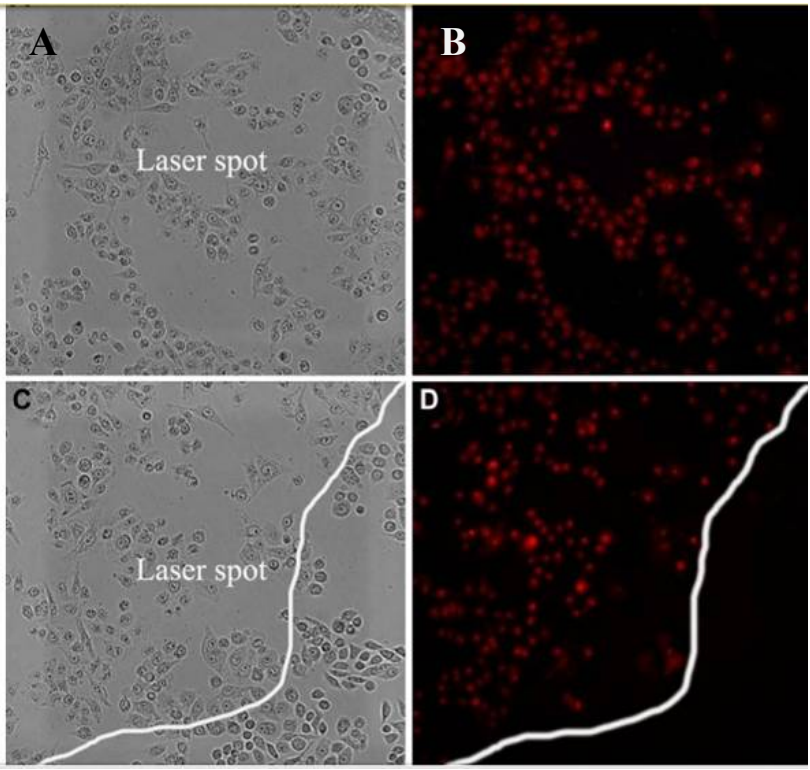

Fig. 2 Photo-thermal therapy effects on MGC 803 cells incubated with $12.5 \mathrm{mM}$ of GNR-SiO2-FA for $24 \mathrm{~h}$ at $37 \mathrm{oC}$ in the dark prior to irradiation for 3 min with $808 \mathrm{~nm}$ laser. (A and B) MGC803 cells on the laser spot center, (C and D) MGC803 cells on the boundary of laser spot. (A and C) Bright field, (B and D) Fluorescence field. Reprinted with permission from Ref. 40.

GNR can also be utilized in optoacoustic imaging (OI) [41]. Several groups have utilized GNRs as contrast agents, and they conclude that GNRs are highly sensitive but poor imaging efficiency. The disadvantage of GNRs is cytotoxicity. Although great efforts have been made to reduce it [42], unless the suitable surface functionalization can be developed, it will be the great challenge of GNRS in clinical application.

\subsection{Gold nanoprisms (GNPrs)}

For anisotropic GNPrs, the optical properties are largely affected by the symmetry and aspect ratio [4344]. GNPrs, a flat and highly symmetrical structure, easily emit ultrasound waves when absorbed the photon

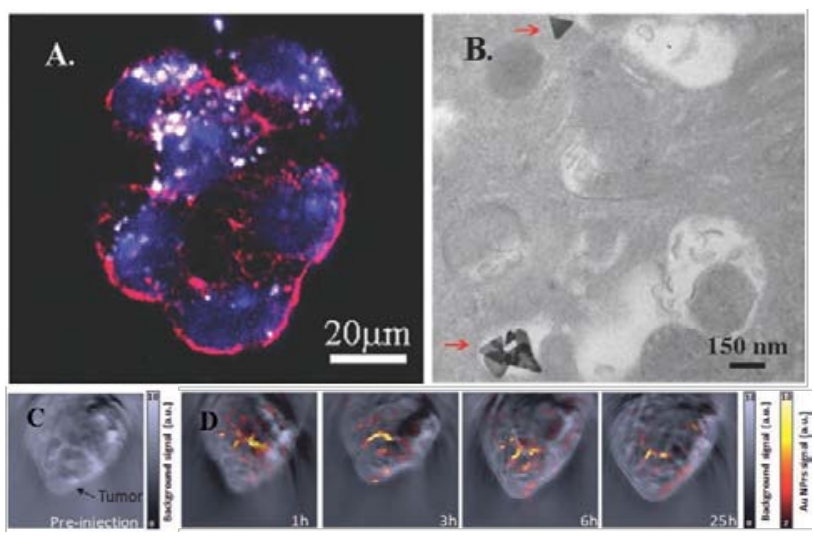

Fig. 4 Two-photon microscopy image of the nanoprisms in straight filaments around the nucleus. (B) the TEM image of HT-29 cells uptaking PEG-coated triangular GNPs (red) in the cytoplasm.; (C) anatomical (pre-injection of the contrast agent) OI, (B) multispectral optoacoustic images of a CD-1 nude mouse bearing a subcutaneous HT29 tumor cell line and injected with GNPrs. Reprinted with permission from Ref. 46.
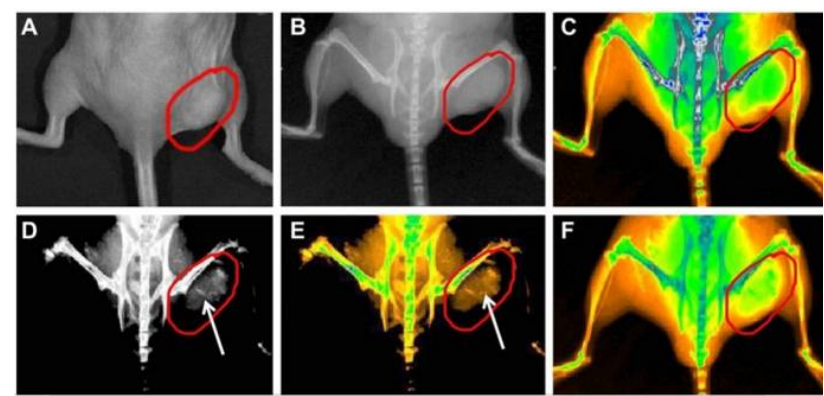

Fig. 3 Real-time in vivo X-ray images after intravenous injection of GNR-SiO2-FA in nude mice at different time points. (A) The photograph of the tumor tissue; (B) the X-ray image at $0 \mathrm{~h}$; (C) the X-ray image at $0 \mathrm{~h}$ (in color); (D) the X-ray image at $12 \mathrm{~h},(\mathrm{E})$ the X-ray image at $12 \mathrm{~h}$ (in color); (F) the X-ray image at $24 \mathrm{~h}$ (in color). (For interpretation of the references to colour in this figure legend, the reader is referred to the web version of this article.) Reprinted with permission from Ref. 40.

and amplify the signal of Multispectral Optoacoustic Tomography (MSOT). Therefore, they have opportunities to visualize cancer by OI and MSOT with the high sensitivity, specificity and spatial resolution [45]. Our group reports PEGylated GNPrs used in tumor imaging both in vitro and in vivo [46]. The PEGylated GNPrs show good biocompatibility and exhibit a surface plasmon band centered at $830 \mathrm{~nm}$, a suitable wavelength for OI purposes. GNPrs are successfully internalized by HT-29 gastrointestinal cancer cells by Two-photoninduced luminescence shown in Fig. 4A and 4B, indicating that GNPrs display distinguished brightness in two-photon luminescence imaging and therefore can also be used as contrast agents. GNPrs are further injected into which mice in order to visualize tumor angiogenesis in gastrointestinal cancer cells shown in Fig. 4C and $4 \mathrm{D}$, indicating that GNPrs stay mostly in the blood flow during the $24 \mathrm{~h}$ and a noticeable amount is retained inside the tumor even after $25 \mathrm{~h}$. Both our in vitro and in vivo results show that PEGylated GNPrs have the capacity to penetrate tumors and provide a high-resolution signal amplifier for optoacoustic imaging. The combination of PEGylated GNPrs and MSOT represents a significant advance for the in vivo imaging of cancers.

De la Fuente group develops a novel and straight forward wet-chemical synthetic route to produce biocompatible GNPrs [43]. The reports method has an unprecedented high yield and small percentage of tiptruncated nanoprisms or nanodisks from the TEM images shown in Fig. 5D and 5E. This method also avoids the use of highly toxic CTAB, which greatly reduce the cytotoxicity of GNPrs. Furthermore, GNPrs are verified as an attractive alternative to those currently in use as photothermal agents. As shown in Fig. 5A, 5B and 5C, the viability of cells loaded with NPRs, cells under irradiation with or without NPRs is evaluated. Bare NPRs, as they result from the synthesis, purified NPRs are derivatized with carboxyl-ended PEG chains and these further derivatized with glucose and the dye TAMRA are proven to be viable up to NPRs concentrations. The result shows that the GNPrs have efficient to kill selectively cells by NIR light. 
http://nanobe.org
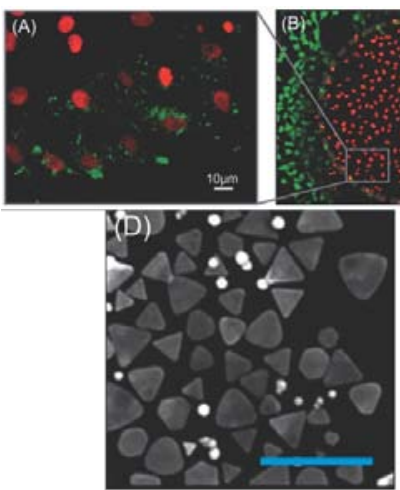

Fig. 5 (A) Merged image of red and green fluorescence channels shows that the viability of irradiated cells is impaired (EthD-1 red staining); (B) red stained cells (EthD-1) correspond to the irradiation spot, whereas cells outside the irradiation area remain viable (Calcein AM, green staining); the dashed line corresponds to the irradiation area; (C) cells outside the irradiation area remain viable (green staining) with NPRs (TAMRA, red staining) in their cytosol; (D, E)SEM images of NPRs with increasing average edge length, scale bar is $500 \mathrm{~nm}$ in all cases. Reprinted with permission from Ref. 43

\subsection{Gold nanostars (GNSs)}

GNSs, that is, star-shaped AuNPs, are a particularly interesting class of plasmonic particles [47]. Their structures contain multiple sharp branches that act as 'lightning rods' to greatly enhance the local EM-field. The small radius of curvature at the ends of the tips gives rise to strongly enhanced fields without the need for particle aggregation [44,48-50]. Individual GNSs exhibit several plasmon resonances in the visible and NIR region that display strong polarization dependence. And this response is correlated with the branch with sharp tips of GNSs. Based on the two important features of GNSs, NIR plasmon and strong near field enhancements at the tips, their applications have been numerous in various biomedical arenas, including PTT, OI. Lately, GNSs are verified to efficiently enhance two-photon photoluminescence (TPL) intensity [51]. Therefore, TPL can be applied to multiphoton microscopy, offering a convenient way to visualize NIR-absorbing gold nanoparticles using NIR excitation, which is preferable for in vivo imaging. Vo-Dinh group uses wheat-germ agglutinin (WGA) functionalized GNSs get the TPL imaging of BT549 breast cancer cells in vitro. In Fig. 6, PEGylated nanostars circulating in the vasculature, examine through a dorsal window chamber in vivo in laboratory mouse studies, demonstrate that gold nanostars can serve as an efficient contrast agent for biological imaging applications.

GNSs are reported to great potential in PTT [52]. VoDinh group also Use SKBR3 breast cancer cells incubated with bare nanostars and observe photothermal ablation within 5 minutes of irradiation (Fig. 7). Meanwhile, on a mouse injected systemically with PEGylated nanostars for 2 days, extravasation of nanostars is observed and localized photothermal ablation is demonstrated on a dorsal window chamber within 10 minutes of irradiation (Fig. 8). These both in vitro and in vivo results of plasmon-enhanced localized hyperthermia are
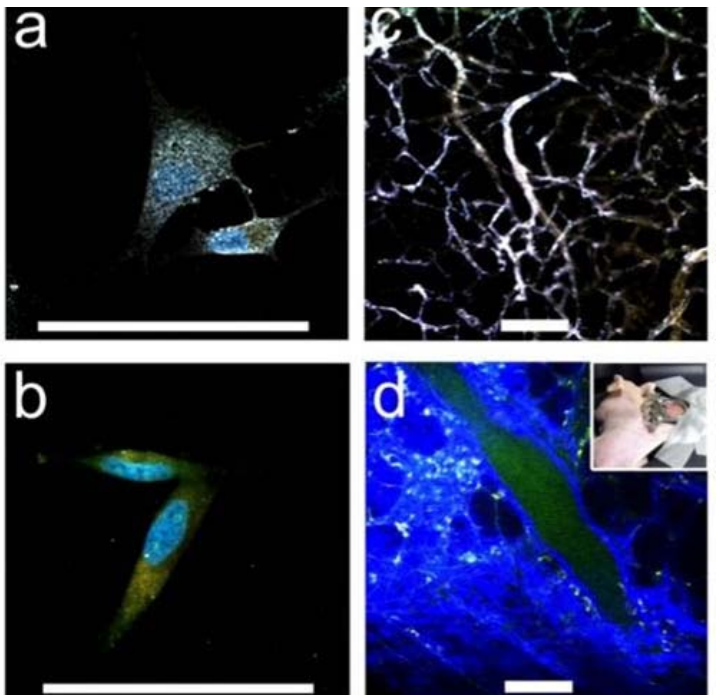

Fig. 6 ((a), (b)) TPL imaging of nanostars S30, WGA-coated (a) versus PEGylated control (b), on BT549 cancer cells. ((c), (d)) TPL imaging through a dorsal window chamber (inset) on a nude mouse. (c) With nanostars, tissue vasculature is visible under 5\% transmission with minimal background fluorescence. (d) Without nanostars, it required $20 \%$ transmission to see vessels. Scale bar: $100 \mu \mathrm{m}$. Reprinted with permission from Ref. 51
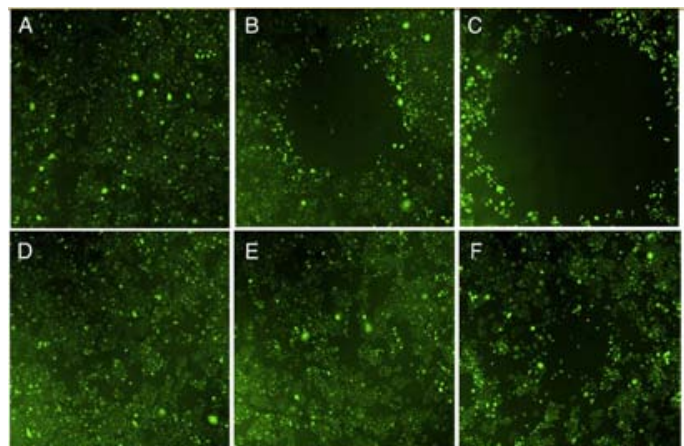

Fig. 7 Fluorescence images of viable SKBR3 cells after the laser treatment. Viable cells appear green by converting non-fluorescent dye into green fluorescence. Empty area indicates successful photothermal ablation. Cells are incubated 1 hour with $0.2 \mathrm{nM}$ nanostars (top) and medium alone (bottom) before the irradiation. Laser irradiation is applied for 1 minute (A, D), 3 minutes (B, E), and 5 minutes $(C, F)$. Image sizes are $3 \times 3 \mathrm{~mm}^{2}$. Reprinted with permission from Ref. 52 .
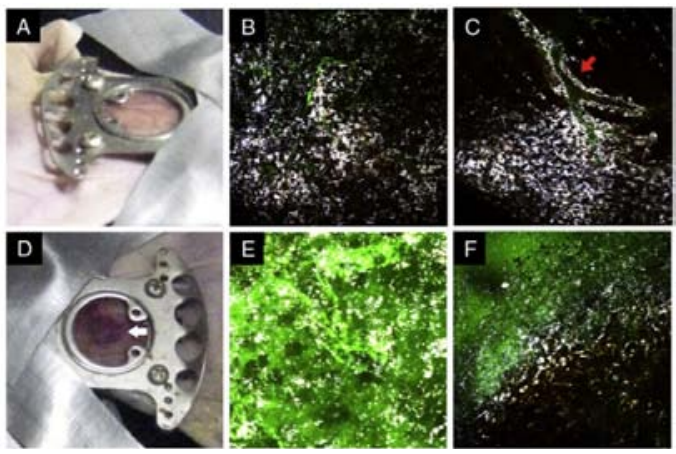

Fig. 8 Photographs and multiphoton microscopy images 48 hours after nanostars infusion before (top) and after (bottom) the laser treatment. Before the irradiation, the window appeared intact and uninflamed (A). Nanostars (white color) scattered in the tissue (B) with some remaining near the perivascular space $(\mathrm{C}$; red arrow). After the laser irradiation, alocalized oozing of blood became visible (D; white arrow). Nanostars distributed more randomly or into clusters. Leakage of FITC-dextran into the tissue is apparent in the irradiated spot (E) but not outside the spot (F). Microscope images are $508 \times 508 \mu^{2}$ and taken under $850-\mathrm{nm}$ $3 \%$ transmission power. Reprinted with permission from Ref. 52 
encouraging and have illustrated the potential of GNSs as efficient photothermal agents in cancer therapy.

\subsection{Gold nanocages (GNCs)}

GNCs are hollow nanostructures with porous walls that can be simply prepared via the galvanic replacement reaction between silver nanocubes and chloroauric acid [53]. GNCs have attractive features such as biocompatibility, easy surface modification for targeting, no potential heavy metal toxicity, a broad range of sizes (35-100 nm), tuning of the localized surface plasmon resonance (LSPR) peak, strong optical absorption in the NIR region [54,55], and encapsulated site-specific drug delivery [56].

Wang's group demonstrate the use of GNCs as a new class of lymph node tracers for noninvasive photoacoustic (PA) imaging of a sentinel lymph node (SLN) in a rat model [57]. As shown in Fig. 9, SLN is noninvasively imaged using the reflection-mode PA imaging with GNCs as tracers. Fig. 9A shows a photograph of the axillae of the rat with hair removed before PA imaging. Fig. 9B is a photograph of the same region with skin and fatty tissue removed, exposing the SLN containing GNCs after all PA images had been captured. Before the injection of GNCs in the forepaw pad, a PA image is acquired as a control (Fig. 9C). For visibility, PA signals beyond the $3 \mathrm{~mm}$ depth are excluded in the image formation. After the injection of GNCs, scanning started immediately to evaluate the migration and accumulation time of the GNCs. PA signals from the SLN are detectable at about 5 min after the injection (Fig. 9D), implying low accumulation of GNCs at this time. As time go by, the contrast at the SLN gradually increase shown in Fig. 9E-G, which means that more GNCs are gradually accumulated by the SLN. Fig. 9H shows the peak accumulation time of GNCs in the SLN at about 140 min after the injection. This research shows that the noninvasive SLN mapping based on GNCs can be beneficial to breast cancer patients. Going forward, by employing surface-modified GNCs, which can bind to specific breast cancer cells, this technique may constitute a noninvasive means of SLNB.

GNCs are also applied in PTT. Xia's group implements the PTT of the immuno GNCs in SK-BR-3 cells [58]. Fig. 10A shows the experimental setup, where cells in the center of a well are irradiated with laser with a spot size of $2 \mathrm{~mm}$. The SK-BR-3 cells are adherent, hence their position is fixed on the surface of the well. It should be pointed out that the laser only irradiated $9.8 \%$ of the cells in each well (note cells are not drawn to scale). In one study, the treated cells are harvested at various times after irradiation to investigate when cells start to respond to the PTT. In Fig. 10B, the cells are irradiated with a laser for $5 \mathrm{~min}$. After that, the cells are returned to a 37 ${ }^{\circ} \mathrm{C}$ incubator for a specific duration of time before the percentage of cellular. Fig. 10C and 10D show the flow cytometry graphs of the cells with and without targeting
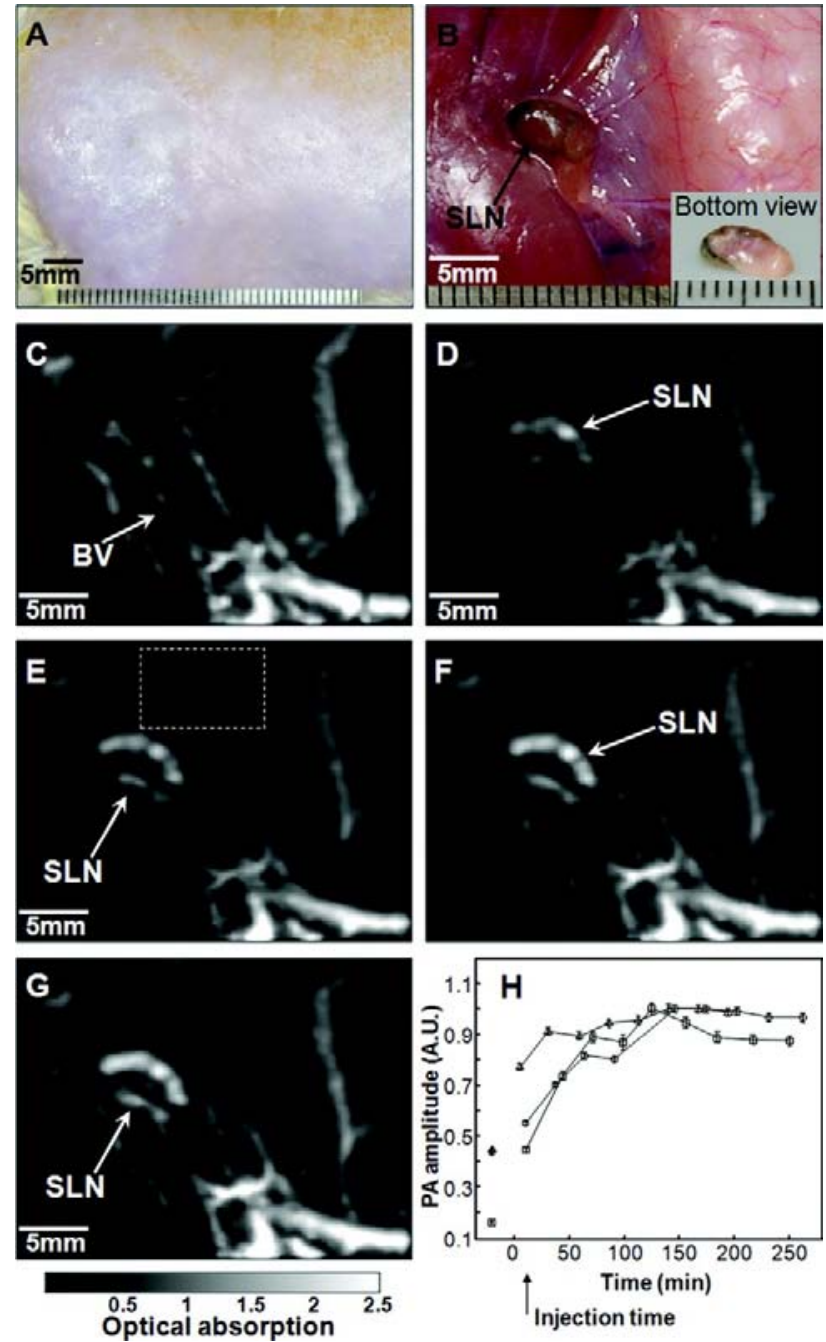

Fig. 9 In vivo noninvasive photoacoustic time-course sagittal MAP images. (A) Photograph of axillary region with hair removed. (B) Photograph with skin and fatty tissue removed after the PA images had been recorded. The dark blue dye lymphatic channel on the bottom of the SLN is shown in the inset. PA images acquired before (C) and after (D - H) the nanocage injection: (D) 5 min (SLN started to appear), (E) $59 \mathrm{~min},(\mathrm{~F}) 140 \mathrm{~min},(\mathrm{G}) 194 \mathrm{~min}$. All images are acquired without signal averaging. $(\mathrm{H})$ Accumulations of nanocages in a SLN over time, in terms of the amplitude changes of PA signals. After the injection, PA signals increased with time, which means gradual accumulations of nanocages. Peak accumulation occurred at $140 \mathrm{~min}$ after the injection. PA signals from the SLN are normalized by those from adjacent blood vessels (the dotted box in Fig. 9E) to minimize the ultrasonic focal effect, and normalized by maximum. Reprinted with permission from Ref. 57.

by the immuno GNCs, respectively, at a harvest time of 3 $\mathrm{h}$ after laser irradiation. The results show that the amount of cellular death can be controlled by both the exposure time and power density of the laser. Cells treated under the same experimental conditions but without immuno GNCs maintained their viability. The efficacy for the immuno GNCs to kill cancer cells can be attributed to their selective targeting and large absorption cross section of NIR light. The results reported here provide insight into how the photothermal response of immuno GNCs can be optimized and controlled. This knowledge is critical to providing effective, noninvasive treatment of cancer in vivo via photothermal therapy. 
http://nanobe.org
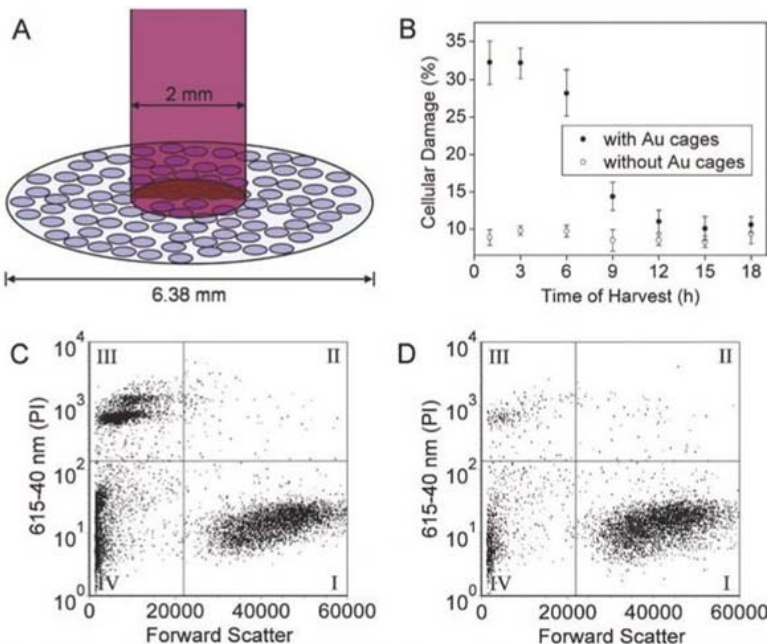

Fig. 10 (A) Illustration depicting the experimental setup used for PTT of SK-BR-3 cells. The cellular growth vessel had a diameter of $6.38 \mathrm{~mm}$. (B) Plots of the percentage of cellular damage versus harvest time when SK-BR-3 cells are irradiated for 5 min at a laser. (C) The flow cytometry graph analyzed to determine the percentage of cellular death after SKBR-3 cells are incubated with immuno GNCs, irradiated for $5 \mathrm{~min}$, the harvested at $3 \mathrm{~h}$ for analysis. (D) Flow cytometry graph obtained from the control experiment in which SK-BR-3 cells are not treated with immuno GNCs but treated to similar laser treatment. For both (C) and (D), the signal in quadrant III corresponds to population of dead cells. Reprinted with permission from Ref. 58.

GNCs have also shown great promise in drug delivery due to their nanostructures with hollow interiors and porous walls and the photothermal effect [28-30]. When the surface of a GNC is covered with a smart polymer, the pre-loaded effector can be released in a controllable fashion using a NIR laser. This system works well with various effectors without involving sophisticated syntheses, and is well suited for in vivo studies owing to the high transparency of soft tissue in the NIR region. Xia's group firstly develops a drug-delivery and controlled release system based on GNCs covered by smart polymers [29]. Fig. 11 shows a schematic of the drug-delivery and controlled release system. The polymer is based on poly( $\mathrm{N}$-isopropylacrylamide) (pNIPAAm) and its derivatives, which can change conformation in response to small variations in temperature .On exposure to a laser beam, the light will be absorbed and converted into heat through the photothermal effect. The heat will dissipate into the surroundings, and the rise in temperature will cause the polymer chains to collapse, exposing the pores on the nanocage and thereby releasing the preloaded effector. When the laser is turned off, heating will immediately cease and the drop in temperature will bring the polymer back to its original, extended conformation, closing the pores and stopping the release. They further extended the controlled release to an in vitro study that involved killing of breast cancer cells with doxorubicin (Dox), a commercial chemotherapeutic drug for breast cancer. The results show, this system has enough efficacy of killing cancer cells.

\section{Conclusion}

In this paper, we summarized the properties and applications of non-spherical AuNPs for cancer imaging 165

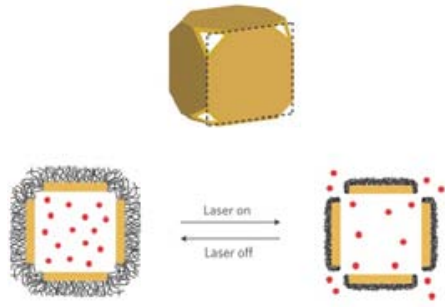

Fig. 11 Schematic illustrating how the system works. A side view of the GNCs is used for the illustration. On exposure to a near-infrared laser, the light is absorbed by the nanocage and converted into heat, triggering the smart polymer to collapse and thus release the pre-loaded effector. When the laser is turned off, the polymer chains will relax back to the extended conformation and terminate the release. Reprinted with permission from Ref. 29.

and therapy. The optical, optothermal and structural properties make non-spherical AuNPs have great application in multi modes of tumor imaging and therapy. We review four typical shapes of non-spherical AuNPs from their application and advantages. GNRs with lesscytotoxicity are used in the dual-mode CT imaging bring novel opportunities in tumor theranostics. They also used to kill cancer cell both in vitro and in vivo. GNPrs are used in OI due to their flat and symmetrical structure. They are also used in PPT and can impair the viability of irradiated cells. GNSs, contain multiple sharp branches, are used in TPL imaging and acted as efficient photothermal agents in cancer therapy. GNCs can be used in PA imaging based on LSPR. Furthermore, they also can used in drug-delivery and controlled release system, which can improve the efficiency of PTT. As this review has clearly shown, different shapes AuNPs have different advantages. We look forward with great optimism to the applications of non-spherical AuNPs in medicine.

\section{Acknowledgment}

This work is supported by the National Key Basic Research Program (973 Project) (No. 2011CB933100), National Natural Scientific Fund (No.81225010,81327002, and 31100717), 863 project of China (2012AA022703), Shanghai Science and Technology Fund (No.13NM1401500 and 11nm0504200), Shanghai Jiao Tong University Innovation Fund for Postgraduates (No. AE340011).

\section{References}

1 Boyle P., Levin B. World Cancer Report. World Health Organization Press; 2008.

2 Jemal A., Siegel R., Xu J., Ward E. Cancer statistics. CA Cancer J. Clin. 2010, 60:277-300.

3 Yu M.K., Park J., Jon S.Y., Targeting strategies for multifunctional nanoparticles in cancer imaging and therapy. Theranostics 2012, 2(1):3-44

4 Zhang L., Gu F.X., Chan J.M., Wang A.Z., Langer R.S., Farokhzad O.C. Nanoparticles in medicine: therapeutic applications and developments. Clin. Pharmacol. Pher. 2008 83:761-769

5 Park K., Lee S., Kang E., Kim K., Choi K., Kwon I.C., New generation of multifunctional nanoparticles for cancer imaging and therapy. Adv. Funct. Mater. 2009,19:1553-1566

6 Choi Y.E., Kwak J.W., Park J.W., Nanotechnology for early cancer detection. Sensors 2010, 10:428-455

7 Cai W., Gao T., Hong H., Sun J., Applications of gold nanoparticles in cancer nanotechnology. Nanotechnol. Sci. Appl. 2008, 1:17-32.

8 Llevot A., Astruc D. Applications of vectorized gold nanoparticles to the diagnosis and therapy of cancer. Chem. Soc. Rev. 2012, 41: 242-257

9 Huang X., Jain P.K., El-Sayed I.H., El-Sayed M.A. Gold 
nanoparticles: interesting optical properties and recent applications in cancer diagnostics and therapy. Nanomedicine, 2007, 2:681-693

10 Mallick K., Wang Z.L. Seed-mediated successive growth of gold particles accomplished by UV irradiation: a photochemical approach for size-controlled synthesis. J. Photoch. Photobio. A 2001,140:75-80

11 Liz-Marzan L. Tailoring surface plasmons through the morphology and assembly of metal nanoparticles. Langmuir 2006, 22:32-41

12 Murphy C.J., Sau T.K., Gole A.M., Odendorff C.J., Gao J., Gou L., Hunyadi S.E., Li T. Anisotropic metal nanoparticles: synthesis, assembly, and optical applications. J. Phys. Chem. B 2005, 109:13857-13870

13 Pérez-Juste J., Pastariza-Santos I., Liz-Marzan L., Mulvaney P., Gold nanorods: Synthesis, characterization and applications. Coord. Chem. Rev. 2005, 249:1807-1901

14 Link S., El-Sayed M. Shape and size dependence of radiative, non-radiative and photothermal properties of gold nanocrystals. Int. Rev. Phys. Chem. 2000, 19, 409-453

15 Huang X.H., El-Sayed I.H., Qian W., El-Sayed M.A. Cancer cell imaging and photothermal therapy in the near-infrared region by using gold nanorods. J. Am. Chem. Soc. 2006, 128:2115-2120

16 Jain P.K., El-Sayed I.H., El-Sayed M.A. Au nanoparticles target cancer. Nano today 2007, 2:18-29

17 Weissleder R. A clearer vision for in vivo imaging. Nat. Biotechnol. 2001, 19:316-317

18 Ahmadi T.S., Logunov S.L., El-Sayed M. A. Picosecond dynamics of colloidal gold nanoparticles. J. Phys. Chem.1996, 100:8053-8056

19 Logunov S.L., Ahmadi T.S., El-Sayed M.A., Khoury J.T., Whetten R.L. Optical absorption spectra of nanocrystal gold molecules. $J$. Phys. Chem. B 1997, 101:3706-3712.

20 Kamat P.V., Shanghavi B. Interparticle electron transfer in metal/ semiconductor composites. picosecond dynamics of CdS-capped gold nanoclusters. J. Phys. Chem. B 1997, 101:7675-7679.

21 Chou C.H., Chen C.D., Wang C.R.C., Highly efficient, wavelengthtunable, gold nanoparticle based optothermal nanoconvertors. J. Phys. Chem. B 2005, 109:11135-11138

22 Huang X.H., El-Sayed M.A. Gold nanoparticles: optical properties and implementations in cancer diagnosis and photothermal therapy. J. Adv. Res. 2010, 1:13-28.

23 Li X.H., Zhang C., Laurent L.G.Y., Chen C.Y. "Smart" nanomaterials for cancer therapy. Sci. China Chem. 2010, 53:2241-2249

24 Tong L., Wei Q.S., Wei A., Cheng J.X. Gold nanorods as contrast agents for biological imaging: optical properties, surface conjugation and photothermal effects. Photochem. Photobiol. 2009, 85:21-32

25 Li Q.Q., Liu F., Lu C., Lin J.M. Aminothiols sensing based on fluorosurfactant-mediated triangular gold nanoparticle-catalyzed luminol chemiluminescence, J. Phys. Chem. C, 2011, 115: 10964-10970

26 Mirkin C.A., Letsinger R.L., Mucic R.C., Storho J.J. A DNA-based method for rationally assembling nanoparticles into macroscopic materials. Nature 1996, 382, 607-609.

27 Von Maltzahn G., Park J.H., Agrawal A., Bandaru N.K., Das S.K., Sailor M.J. Computationally guided photothermal tumor therapy using long-circulating gold nanorod antennas. Cancer Res. 2009, 69(9):3892-900.

28 Chamberland D.L., Agarwal A., Kotov N., Fowlkes J.B., Carson P.L., Wang X. Photoacoustic tomography of joints aided by an Etanerceptconjugated gold nanoparticle contrast agent-an ex vivo preliminary rat study. Nanotechnology 2008, 19, 095101.

29 Yavuz M.S., Cheng Y.Y., Chen J.Y., Cobley C.M.,Zhang Q., Rycenga M., Xie J.W., Kim C., Song K.H.,. Schwartz A.G, Wang L.H.V., Xia Y.N. Gold nanocages covered by smart polymers for controlled release with near-infrared light. Nat. Mater. 2009, 8:935-939.

30 Moon G.D., Choi S.W., Cai X., Li W.Y., Cho E.C., Jeong U., Wang L.V., Xia Y.N. A new theranostic system based on gold nanocages and phase-change materials with unique features for photoacoustic imaging and controlled release. J. Am. Chem. Soc. 2011 , 133:4762-4765

31 Nikoobakht B., El-Sayed M.A. Preparation and growth mechanism of gold nanorods (NRs) using seed-mediated growth method. Chem. Mater. 2003, 15:1957-1962.

32 Thomas K.G., Barazzouk S., Ipe B.I., Joseph S.T.S., Kamat P.V. Uniaxial plasmon coupling through longitudinal self-assembly of gold nanorods. J. Phys. Chem. B 2004 108:13066-13068
33 Li X. Qian J., He S.L. Impact of the self-assembly of multilayer polyelectrolyte functionalized gold nanorods and its application to biosensing. Nanotechnology 2008, 19:355501-355508

34 Jana N.R., Gearheart L., Murphy C.J. Wet chemical synthesis of high aspect ratio cylindrical gold nanorods. J. Phys. Chem. B 2001, 105:4065-4067.

35 Gómez-Graña S., Hubert F., Testard F., Guerrero-Martínez A., Grillo I., Liz-Marzán L.M. . Surfactant (Bi) layers on gold nanorods. Langmuir 2012, 28:1453-1459.

36 Zhang J.J., Liu Y.G., Jiang L.P. Synthesis, characterizations of silica-coated gold nanorods and its applications in electroanalysis of hemoglobin. Electrochem. Comm. 2008,10: 355-358.

37 Murphy C.J., Gole A.M., Stone J.W. Gold nanoparticles in biology: beyond toxicity to cellular Imaging. Acc. Chem. Res. 2008, 41:1721-1730.

38 Alkilany A.M., Nagaria P.K., Hexel C.R., Cellular uptake and cytotoxicity of gold nanorods: molecular origin of cytotoxicity and surface effects. Small 2009, 5:701-708.

39 Li Z.M., Huang P., Zhang X.J., Lin J., Yang S., Liu B., Gao F., Xi P., Ren Q.S., Cui D.X. RGD-conjugated dendrimer-modified gold nanorods for in vivo tumor Targeting and photothermal therapy. Mol. Pharm. 2010, 7 (1): 94-104

40 Huang P., Bao L., Zhang C.L., Lin J., Luo T., Yang D.P., He M., Li Z.M., Gao G., B. Gao, S. Fu, D.X. Cui, Folic acid-conjugated Silicamodified gold nanorods for X-ray/CT imaging-guided dual-mode radiation and photo-thermal therapy. Biomaterials 2011, 32: 9796-9809

41 Franchini M.C., Ponti J., Lemor R. Polymeric entrapped thiol-coated gold nanorods: cytotoxicity and suitability as molecular optoacoustic contrast agent. J. Mater. Chem. 2010, 20:10908-10914.

42 Zhang J.J., Liu Y.G., Jiang L.P., Zhu J.J.Synthesis, characterizations of silica-coated gold nanorods and its applications in electroanalysis of hemoglobin, Electrochem. Comm. 2008, 10:355-358

43 Pelaz B., Grazu V., Ibarra A., Magen C., del Pino P., de la Fuente J.M. Tailoring the synthesis and heating ability of gold nanoprisms for bioapplications. Langmuir 2012, 28:8965-8970

44 Millstone J.E.; Park S., Shuford K.L., Qin L., Schatz G.C., Mirkin C.A. Observation of a quadrupole plasmon mode for a colloidal solution of gold nanoprisms. J. Am. Chem. Soc. 2005, 127: $5312-5313$

45 Insana M.F., Pellot-Barakat C., Sridhar M., Lindfors K.K. Viscoelastic imaging of breast tumor microenvironment with ultrasound. J. Mammary Gland Biol. 2004, 9:393-404

46 Bao C.C., Beziere N., del Pino P., Pelaz B., Estrada G., Tian F., Ntziachristos V., de la Fuente J.M., Cui DX. Gold nanoprisms as optoacoustic signal nanoamplifiers for in vivo bioimaging of gastrointestinal cancers. small 2013, 9:67-74

47 Hrelescu C., Sau T.K., Rogach A.L., Jäckel F., Laurent G., Douillard L., Charra F.. Selective excitation of individual plasmonic hotspots at the tips of single gold nanostars, Nano Lett. 2011, 11:402-407

48 Pastoriza-Santos I., Liz-Marzan L.M. Synthesis of silver nanoprisms in DMF. Nano Lett. 2002, 2:903-905.

49 Kim F., Connor S., Song H., Kuykendall T., Yang P., Platonic gold nanocrystals. Angew. Chem. Int. Ed. 2004, 43:3759-3763.

50 Pastoriza-Santos I., Liz-Marzan L.M. Colloidal silver nanoplates. State of the art and future challenges. J. Mater. Chem. 2008, 18: 1724-1737.

51 Yuan H., Khoury C.G., Hwang H., Wilson C.M., Grant G.A., Vo-Dinh T. Gold. nanostars: surfactant-free synthesis, 3D modelling, and two-photon photoluminescence imaging. Nanotechnology 2012, 23:075102

52 Yuan H., Khoury C.G., Wilson C.M., Grant G.A. Bennett A.J., Vo-Dinh T., In vivo particle tracking and photothermal ablation using plasmon-resonant gold nanostars. Nanomed-Nanotechnol. 2012,8:1355-1363

53 Chen J.Y., Yang M.X., Zhang Q., Cho E.C., Cobley C.M., Kim C., Glaus C., Wang L.V., Welch M.J., Xia Y.N., Gold nanocages: a novel class of multifunctional nanomaterials for theranostic applications. Adv. Funct. Mater. 2010, 20:3684-3694

54 Chen J., Saeki F., Wiley B.J., Cang H., Cobb M.J., Li Z.Y., Au L., Zhang H., Kimmey M.B., Li X., Xia, Y.N. Gold nanocages: bioconjugation and their Potential use as optical imaging contrast agents. Nano Lett. 2005, 5:473-477. 
http://nanobe.org

55 Hu M., Chen J.Y., Li Z.Y., Au L., Hartland G. V.; Li X. D., Marquez M., Xia Y.N. Gold nanostructures: engineering their plasmonic properties for biomedical applications. Chem. Soc. ReV. 2006, 35:1084-1094.

56 Sun Y., Xia Y.N. Mechanistic study on the replacement reaction between silver nanostructures and chloroauric acid in aqueous medium. J. Am. Chem. Soc. 2004, 126:3892-3901.

57 Song K.H., Kim C., Cobley C.M., Xia Y.N., Wang L.V. Near-infrared gold nanocages as a new class of tracers for photoacoustic sentinel lymph node mapping on a rat model. Nano Lett. 2009, 9:183-188
58 L. Au, D.S. Zheng, F. Zhou, Z.Y. Li, X.D. Li, Y.N. Xia. A quantitative study on the photothermal effect of immuno gold nanocages targeted to breast cancer cells. ACS Nano 2008, 2:1645-1652

Copyright:(c) 2013 Y.S. Chen and D.X. Cui. This is an openaccess article distributed under the terms of the Creative Commons Attribution License, which permits unrestricted use, distribution, and reproduction in any medium, provided the original author and source are credited. 